$\$$ Research Square

\title{
Correlation between sarcopenia and arteriosclerosis in elderly community dwellers: A multicenter study
}

\author{
Ting Sun \\ Bengbu Medical College https://orcid.org/0000-0002-7961-5395 \\ Zuchang Ma \\ Institute of Science and Technology Chinese Academy of Sciences \\ Lisheng Gao \\ University of the Chinese Academy of Sciences \\ Hui Xie ( $\square$ hui2122@hotmail.com) \\ Bengbu Medical College
}

\section{Research article}

Keywords: Arteriosclerosis, Sarcopenia, Handgrip stretch, Pulse wave

Posted Date: August 20th, 2020

DOI: https://doi.org/10.21203/rs.3.rs-58740/v1

License: (c) (i) This work is licensed under a Creative Commons Attribution 4.0 International License. Read Full License 


\section{Abstract \\ Background}

Evidence suggests that arteriosclerosis and sarcopenia (decreased muscle mass) share some of their many causes. However, after controlling for confounding factors, it is unclear whether the presence of arteriosclerosis correlates with sarcopenia. The aim of this paper is to explore whether sarcopenia is associated with arteriosclerosis.

\section{Methods}

A total of 2511 elderly subjects from six Chinese community health service centers in Anhui province were surveyed through an e-health promotion system to collect basic data and measurements of brachial-ankle pulse wave (baPWV), body composition, and handgrip strength (HGS). Pearson's correlation and binary logistic regression analyses were performed to identify associations between sarcopenia and high baPWV.

\section{Results}

The prevalence rates of sarcopenia were $12.9 \%$ in men and $15.3 \%$ in women according to the 2019 standard of Asian Working Group for Sarcopenia. Among subjects with high baPWV, the proportion of sarcopenia was higher compared to those with normal baPWV (men: $17.7 \%$ vs $3.7 \%$; women: $20.4 \%$ vs $4.9 \%$, both $P<0.001)$. Binary logistic regression analysis revealed that sarcopenia was associated with high baPWV $(P<$ 0.0001 , odds ratio 1.619) after adjusting for confounding factors. HGS slightly and negatively correlated with baPWV (-0.19 in men and - 0.18 in women).

\section{Conclusions}

The intertwined pathophysiological mechanisms shared by arteriosclerosis and sarcopenia are potential targets for future interventions to reduce morbimortality in subjects with both disorders. Upcoming prospective studies and clinical trials are expected to advance these findings.

\section{Background}

Sarcopenia is a geriatric syndrome characterized by loss of muscle mass, muscle strength, and physical function [1]. The Asian Working Group for Sarcopenia (AWGS) 2014 consensus defined sarcopenia as an age-related illness with the symptoms mentioned above [2]. Increasing evidence demonstrates that sarcopenia is associated with many negative outcomes such as functional disability, poor quality of life, falls, and increased mortality; the condition has complex interactions with many chronic diseases such as cancer and diabetes [1]. The incidence of sarcopenia increases with age but varies strongly depending on the use of varying definitions and related cut-off points. At present, there are two internationally recognized evaluation criteria: one from the AWGS [2] and another from the European Working Group on Sarcopenia in Older People (EWGSOP) [3]. Both guidelines recommend the presence of both low muscle mass and low muscle function (strength or physical performance) for sarcopenia diagnosis, but there are slight differences in cut-off points [2, 3]. According to the AWGS definition, the prevalence in Asian communitydwelling elder adults varies between $8.2 \%$ and $25.7 \%$ [4-14]

Pulse wave velocity (PWV) is proportional to arterial wall firmness [15] and has two principal functions. First, PWV can indicate an elevated risk of progressive organ dysfunction (e.g., high blood pressure or renal failure). Second, PWV can facilitate a diagnosis of heart disease [16]. According to the Japanese Guidelines for Noninvasive Vascular Function Test (JCS2013), lifestyle changes should be suggested for subjects with a brachialankle PWV (baPWV) of $1400 \mathrm{~cm} / \mathrm{s}$, which equates to a moderate Framingham risk score. At this level, there is a high risk of developing high blood pressure [17].

Arteriosclerosis and loss of muscle mass (decreased muscle function) share some cause, including age-related changes, hypokinesia, nutrition status, inflammation, vitamin-D level, insulin resistance, low bone density, and hormonal imbalance, which complicates the relationship between arteriosclerosis and sarcopenia [18]. For example, we know that the growth hormone (GH) and insulin-like growth factor-I (IGF-1) levels decline with age and are associated with loss of mobility and muscle mass, as well as cardiovascular complications. GH and IGF-1 mediate a variety of protein synthesis and degradation pathways that play important roles in increasing and rebuilding muscle fiber, strength and function. Through its receptor, IGF-1 exerts a variety of physiological and pathophysiological functions on the vasculature, including stimulation of smooth muscle cell (SMC) proliferation, migration, and apoptosis. Decreases in IGF-1 may change the structure of vascular SMCs, which will lead to hypertension and other diseases, and it can also promote plaque formation, contributing to atherosclerosis [19].

Here we controlled for age as a confounding factor to determine if there is a correlation between arteriosclerosis and sarcopenia. We assessed participants ( $\geq 60$ years) who visited Chinese community health service centers located in Anhui province (Hefei, Bengbu, and Chuzhou) for both 
sarcopenia (using AWGS criteria) and baPWV. This study aimed to investigate the associations between the incidence of sarcopenia (including muscle mass-related parameter and handgrip strength [HGS]) and high baPWV.

\section{Methods}

\section{Study population}

The cross-sectional data were collected from a community-based study used to identify factors that contribute to non-communicable chronic diseases. Artificial intelligence methods were applied to examine the impact of health promotions. The data include anthropometric and biochemical parameters, heart function, lifestyle, illnesses, family medical history, and mental health. An electronic health promotion system was used to collect data on an annual basis for these dwellers.

Residents were offered the chance to participate in the investigation through six community health service centers located three cities in Anhui province (Bengbu, Chuzhou, and Hefei). The total number of participants aged 18 or older was 4529 . They were surveyed from June 2018 to January 2020. Three exclusion criteria were applied: 1896 individuals not aged 60 or older were excluded, 59 participants without baPWV data were excluded, and 63 participants without HGS data were excluded. This left 2511 participants with sufficient data, including 1475 females and 1036 males. The mean age of the participants was 65.88 (SD 5.81). Each participant was asked to provide written, informed consent for the use of their data for this research. The Ethics Committee of Bengbu Medical College approved the study protocol (Anhui, China; no. 2018045).

\section{Anthropometric data}

Three anthropometric measures were taken for each participant: height, weight, and waist circumference (WC). Height and WC were measured using a steel measuring tape, and weight was assessed with a bioelectric impedance analyzer (BIA, Model BX-BCA-100; Institute of Intelligent Machines, Hefei, China). Weight and height were used to calculate BMI.

\section{Body composition measurements}

Participants fasted from food and water in the 3 hours prior to measurement. They were told to remove their shoes and socks and position themselves on the BIA machine. Electrodes were applied to their extremities and the participants were told to lift both arms and touch the electrodes. Skeletal muscle mass (SMM) was calculated according to the formula SMM (kg) $=0.566$ * FFM [20]. where FFM denotes the fat free mass computed by the BIA. Appendicular muscle mass index (AMI) was calculated using the formula AMI = (ASM)/height2 [1], where ASM is appendicular skeletal muscle mass.

\section{HGS measurements}

HGS (defined here as the maximal HGS of the dominant hand) was measured using a spring-type dynamometer (TSN100/200-WL, Ti Shi Neng Sports Technology company, Beijing, China). While standing and extending their arms at their sides, participants were told to squeeze the dynamometer as hard as possible for up to 3 seconds. They were asked to do this three times with 30 -seconds rests between each attempt.

\section{Measurement of baPWV and definition of high PWV}

An IIM-AS-100 system (Institute of Intelligent Machines) was used to record baPWV (m/s). It uses oscillation to record bilateral brachial and posterior tibial-artery pressure waveforms with cuffs applied to the participants' arms and ankles. The baPWV for each arterial segment is computed according to the formula baPWV = path length/time.

The Japanese Guidelines for Noninvasive Vascular Function Test considers a baPWV >14 m/s as unhealthy and suggestive of an elevated risk of high blood pressure in untreated normotensive individuals [17].

\section{Definition of sarcopenia}

Based on the AWGS criteria (2019) [2], sarcopenia is characterized as the incidence of low muscle mass, low muscle strength, and/or low physical performance. Here, low muscle mass and low muscle strength were used. Low muscle mass was defined as an AMl $<7.0 \mathrm{~kg} / \mathrm{m}^{2}$ in males and $<5.7$ $\mathrm{kg} / \mathrm{m}^{2}$ in females. Low muscle strength was defined as a HGS $<26 \mathrm{~kg}$ for males and $<18 \mathrm{~kg}$ for females.

\section{Data collection}

All physical examinations were performed by trained medical staff or medical postgraduate students according to standardized procedures. Participants were questioned regarding health-related behaviors including cigarette and alcohol consumption and physical activity levels. For cigarette consumption, total smoking during the subject's lifetime was calculated based on the quantity of cigarettes that were smoked and the weekly frequency; this was extended to consumption before quitting for former smokers. The amounts of alcohol in one bottle of the most popular alcoholic beverages in Anhui province are as follows: beer (500 ml, 3.2\% alcohol), $17.5 \mathrm{~g}$; white liquor (450 ml, $42 \%$ alcohol), $210 \mathrm{~g}$; and wine (750 
$\mathrm{ml}, 13.5-14 \%$ alcohol), $97.5 \mathrm{~g}$. Daily alcohol consumption was calculated using these values. When data for cigarette and alcohol consumption were missing, a value of zero was assigned. Subjects were questioned about the types of physical activities in which they engaged, the duration of activity (minutes), and the frequency (per week). According to activity codes and metabolic equivalent (MET) intensities in the Compendium of Physical Activities, physical activity time was determined as minutes/MET/day, and missing values were assigned the median value. The remaining data were collected through self-report questionnaires, and missing values were assigned a value of zero.

\section{Statistical analyses}

SPSS v23.0 software (IBM, Armonk, NY, USA) was utilized for data analyses. All continuous variables are expressed as mean \pm SD. The significance of differences in baseline characteristics between participants with and without sarcopenia were examined using Student's t-tests for continuuus variable and Pearson's chi-squared tests for discontinuous variable. Significance test calculations were stratified by gender. Student's t-tests were also used to assess differences in sarcopenia rates among participants with normal and high baPWV.

HGS/AMI and baPWV were investigated with Pearson's correlations. Whether Age and prevalence of sarcopenia independently correlated with high PWV were determined using binary logistic regression models that were adjusted for smoking and drinking levels, sleep issues, kidney/heart disease, high blood pressure, and physical activity time.

\section{Results}

Among the 2511 subjects (1036 [41.3\%] women], 1589 [63.3\%] 60-69 year-olds, with a mean age of 68.55 years [range, 60-94 years]). Table 1 shows the sample distributions according to sarcopenia status and stratified by sex. A total of 134 (12.9\%) men and $226(15.3 \%)$ women were classified as having sarcopenia based on AMI and HGS. Few significant differences were observed among subjects with or without sarcopenia for weight, height, BMI, WC, cigarette/alcohol consumption, physical activity/sedentary time, sleep disorders, kidney/cardiovascular disease, hypertension, or AMI for either gender. Exceptions include age, height, and cigarette/alcohol consumption in women, sleep disorder, kidney/cardiovascular disease, and hypertension in men, for which slight differences were observed $($ all $P<0.05)$. 
Table 1

Characteristics of subjects with or without sarcopenia stratified by gender

\begin{tabular}{|c|c|c|c|c|c|c|c|}
\hline & & Male & $n=1036(41.3 \%)$ & & Female & $\begin{array}{l}n=1475 \\
(58.7 \%)\end{array}$ & \\
\hline & $\begin{array}{l}\text { Overall sample } \\
(\mathrm{N}=2511)\end{array}$ & $\begin{array}{l}\text { Non-sarcopenia } \\
(\mathrm{n}=902)\end{array}$ & $\begin{array}{l}\text { Sarcopenia } \\
(n=134)\end{array}$ & $\begin{array}{l}P \\
\text { value }\end{array}$ & $\begin{array}{l}\text { Non- } \\
\text { Sarcopenia } \\
(n=1249)\end{array}$ & $\begin{array}{l}\text { Sarcopenia } \\
(n=226)\end{array}$ & $\begin{array}{l}\mathrm{P} \\
\text { value }\end{array}$ \\
\hline Age, years & 68.55(5.81) & $69.24(6.1)$ & $70.19(6.36)$ & 0.093 & 67.85(5.43) & 68.73(5.83) & 0.028 \\
\hline Weight, kg & $64.28(10.31)$ & 69.35(10.02) & $69.17(9.82)$ & 0.844 & 60.77(8.91) & $60.53(9.24)$ & 0.706 \\
\hline Height, cm & $162.15(7.67)$ & 168.7(5.77) & $168.23(5.21)$ & 0.374 & 157.71(5.26) & $156.95(4.71)$ & 0.042 \\
\hline BMI, kg/m² & 24.41(3.31) & 24.34(3.09) & 24.43(3.22) & 0.763 & 24.44(3.45) & $24.55(3.46)$ & 0.64 \\
\hline $\begin{array}{l}\text { Waist } \\
\text { circumference, cm }\end{array}$ & $89.17(9.71)$ & $90.68(9.35)$ & $90.22(8.22)$ & 0.592 & $88.16(9.97)$ & $88.09(9.66)$ & 0.922 \\
\hline $\begin{array}{l}\text { Cigarette } \\
\text { consumption, } \\
\text { cigarettes/lifetime }\end{array}$ & $28,246.58(1778.67)$ & $63,287.03(4048.14)$ & $76,470.22(11,200.49)$ & 0.246 & $97.6(39.6)$ & $96.9(14.56)$ & 0.015 \\
\hline $\begin{array}{l}\text { Alcohol } \\
\text { consumption, } \\
\text { g/day }\end{array}$ & $3.46(0.29)$ & $7.91(0.74)$ & $6.11(1.48)$ & 0.372 & $0.57(0.12)$ & $0.11(0.08)$ & 0.002 \\
\hline $\begin{array}{l}\text { Physical activity } \\
\text { time, } \\
\text { min/MET/day }\end{array}$ & 257.27(176.18) & 261.87(6.24) & 238.27(14.15) & 0.167 & $258.88(4.93)$ & $241.28(9.58)$ & 0.103 \\
\hline $\begin{array}{l}\text { Sedentary time, } \\
\mathrm{min} / \text { day }\end{array}$ & $38.21(1.48)$ & $43.17(2.61)$ & $55.31(7.91)$ & 0.098 & $35.54(1.98)$ & $33.98(4.47)$ & 0.753 \\
\hline $\begin{array}{l}\text { Sleep disorder, } \\
\mathrm{n}(\%)\end{array}$ & $333(13.3)$ & $182(14.6)$ & $46(20.4)$ & 0.027 & $83(9.2)$ & 22(16.4) & 0.010 \\
\hline $\begin{array}{l}\text { Kidney disease, } n \\
(\%)\end{array}$ & $18(0.7)$ & $5(0.4)$ & $5(2.2)$ & 0.002 & $6(0.7)$ & $2(1.5)$ & 0.307 \\
\hline $\begin{array}{l}\text { Cardiovascular } \\
\text { disease, n (\%) }\end{array}$ & $284(11.3)$ & 137(17) & 43(19) & 0.001 & $89(9.9)$ & $15(11.2)$ & 0.633 \\
\hline Diabetes, n (\%) & $348(13.9)$ & $146(11.7)$ & 41(18.1) & 0.007 & $129(14.3)$ & $32(23.9)$ & 0.004 \\
\hline $\begin{array}{l}\text { Hypertension, n } \\
(\%)\end{array}$ & $927(36.9)$ & 408(35) & $100(45.5)$ & 0.003 & $356(42)$ & $63(49.2)$ & 0.126 \\
\hline baPWV, m/s & $12.68(7.46)$ & $12.15(7.73)$ & $17(4.15)$ & $\begin{array}{l}P< \\
0.0001\end{array}$ & 11.84(7.62) & 16.84(3.93) & $\begin{array}{l}P< \\
0.0001\end{array}$ \\
\hline $\begin{array}{l}\text { Handgrip } \\
\text { strength, kg }\end{array}$ & 16.57(9.8) & $21.18(6.2)$ & 19.02(13.05) & 0.002 & 14.83(7.11) & 13.72(3.81) & 0.001 \\
\hline $\mathrm{AMI}, \mathrm{kg} / \mathrm{m}^{2}$ & $4.57(0.8)$ & $5.29(0.63)$ & $5.4(0.68)$ & 0.059 & $4.04(0.38)$ & $4.08(0.39)$ & 0.199 \\
\hline \multicolumn{8}{|c|}{ *Continuous variables are presented as mean (standard deviation), and discontinuous variables are presented as number and percentage. } \\
\hline $\begin{array}{l}\text { tP values for the dif } \\
\text { tests. }\end{array}$ & rence between sarco & nia and non-sarcoper & ia groups stratified by $\mathrm{g}$ & der were & letermined wit & -tests and Chi & quared \\
\hline
\end{tabular}

Comorbid diabetes was more common in subjects with sarcopenia for both men and women, with rates of $18.1 \%$ and $23.9 \%$, respectively $(P<$ 0.01). baPWVs were also increased in subjects with sarcopenia (17 vs 12.15 for men, 16.84 vs 11.84 for women; both $P<0.0001$ ). The opposite situation was observed for HGS (19.02 vs 21.18 for men, 13.72 vs 14.83 for women, both $\mathrm{P}=0.01$ ).

Among subjects with high baPWV, the proportion of sarcopenia was higher than in the normal baPWV group (men: 17.7\% [ $\mathrm{n}=122]$ vs 3.7\% [ $=12$ ]; women: $20.4 \%$ [ $=202$ ] vs $4.9 \%$ [ $=24]$; both $P$ < 0.001) (Fig. 2). The Pearson's correlation value between AMI and baPWV was not significant (P > 0.05), whereas there was a small but significant negative correlation between HGS and baPWV. Correlation coefficients were - 0.19 and - 0.18 in men and women, respectively (Fig. 3). 
Table 2 shows the potential main predictors of high baPWV (age, sex, and sarcopenia) using binary logistic regression analyses after adjustment of confounding factors including cigarette/alcohol consumption, sleep disorders, kidney/cardiovascular disease, hypertension, and physical activity time. Sarcopenia was associated with an increased likelihood of high baPWV (odds ratio $=1.635,95 \%$ confidence interval $=1.437-7.413$ ). Neither age nor sex was associated with high baPWV. The model explained $45.9 \%$ of the variables.

Table 2

Binary regression analysis of sarcopenia and high baPWV

\begin{tabular}{|c|c|c|c|}
\hline Variables & OR & $95 \% \mathrm{Cl}$ & $P$ value \\
\hline Age 1 ( $70-79$ years vs $60-69$ years ) & -0.596 & $0.359-0.849$ & 0.359 \\
\hline Age 2 ( $\geq 80$ years s $60-69$ years ) & -0.089 & $0.612-1.368$ & 0.665 \\
\hline Sex (male vs female) & 0.12 & $0.947-1.343$ & 0.178 \\
\hline Sarcopenia & 1.619 & $1.437-7.413$ & $<0.0001$ \\
\hline
\end{tabular}

*The module adjusted for confounding factors including cigarette/alcohol consumption, sleep disorder,

kidney/cardiovascular disease, hypertension, and physical activity time. Abbreviations: baPWV, brachial-ankle pulse wave velocity; Cl, confidence interval; OR, odds ratio.

\section{Discussion}

This study extensively explored the relationship between sarcopenia and arteriosclerosis in a sample of community dwellers $\geq 60$ years. The proportion of sarcopenia was higher in subjects with high baPWV compared to those with normal baPWV. There was a small but significant negative correlation between HGS and baPWV, but we did not observe an association between AMI and baPWV. After adjusting for multiple confounders, the presence or sarcopenia was associated with an increased likelihood of high PWV.

We found that sarcopenia was highly prevalent in both genders of elderly Chinese community dwellers (men $12.9 \%$ vs women $15.3 \%$ ), but women were more susceptible than men. This finding is consistent with previous results. We identified 11 studies addressing the prevalence of sarcopenia in elderly community-dwelling subjects. Of these, three were conducted in Japan [4-6], seven in the Chinese mainland [7-13], and one in Taiwan[4-6]. All of these applied the 2014 EWGSOP criteria to define sarcopenia and found that the prevalence ranged from $8.2-25.7 \%$.

Previous reports have described significant and negative associations between arterial stiffness and HGS in nonhypertensive women in both India and Japan [21]. However, a cross-sectional study showed the opposite result; a total of 400 Dutch men aged 40-80 years were surveyed and showed that HGS was not associated with arterial stiffness after adjusting for relevant confounders such as age, educational level, comorbidity, antihypertensive drug use, and smoking and alcohol habits [22]. This discrepant result deserves further study. In terms of the relationship between skeletal muscle and PWV, Abbatecola et al. conducted a large cohort study and concluded that PWV was independently and inversely associated with skeletal muscle metrology in Americans aged 70 to 90 years [23]. The variability might be partially explained by different study designs and populations.

We found that participants with high PVW tended to be more likely to have sarcopenia (men: $17.4 \%$, women: $20.4 \%$ ) than those with normal PVW (men: 3.7\%, women: 4.9\%). Binary logistic regression revealed that sarcopenia was associated with an increased likelihood of high PWV. This finding is consistent with the report by Hida et al. In a study of 335 Japanese subjects with a mean age of 64.9, risk factors for arteriosclerosis (hypertension and elevated cholesterol) were higher in subjects with sarcopenia than those without after adjusting for age, sex, and BMI ( $<0.05$ ) [24].

The relationship between arteriosclerosis and the occurrence and development of arteriosclerosis is complicated. One theory posits that atherosclerosis develops following molecular and cellular inflammation, which is also an underlying factor [25]. Inflammatory factors such as tumor necrosis factor and interleukin 6 are important mediators that stimulate protein catabolism, inhibit muscle synthesis, and promote muscle atrophy [26]. We found that community-dwelling subjects with sarcopenia always showed elevations in blood pressure, serum total cholesterol, low-density lipoprotein cholesterol, and high-sensitivity C-reactive protein (hs-CRP) [24]. High hs-CRP was also an independent risk factor for sarcopenia. As a predictor of cardiovascular events, hs-CRP may be associated with physical function, and high levels have been experimentally associated with an increased risk of reduced muscle strength [27].

Another possible mechanism is insulin resistance (IR), a component of metabolic syndrome, that is associated with obesity, hypertension, dyslipidemia, and arteriosclerosis. IR is a common intermediate or confounding factor between the conditions of myasthenia gravis and arteriosclerosis [28]. IR and the associated increase in advanced glycation end products can lead to micro- and macrovascular complications that directly affect skeletal muscle nutrition [29]. 
The third possible mechanism is muscle factor levels. Skeletal muscle produces and releases substances known as muscle factors that can regulate different metabolic processes. Skeletal muscle-derived factors such as irisin and follistatin-like-1 affect arteriosclerosis, and follistatin-like1 blocks vascular disease progression by inhibiting vascular smooth muscle growth [30]. The relationship between atherosclerosis and sarcopenia requires more research and further investigation to elucidate the complex underlying mechanisms.

\section{Conclusions}

The objective of this study was to investigate a possible association between arteriosclerosis and sarcopenia by measuring HGS and musclerelated parameters. The findings show that baPWV is associated with sarcopenia. The shared pathophysiological mechanisms of arteriosclerosis and sarcopenia are potential targets for interventions to reduce morbimortality in subjects with these conditions. Future prospective studies and clinical trials are expected to advance the applicability of these findings.

\section{Abbreviations}

baPWV, brachial-ankle pulse wave velocity; BMI, body mass index; MET, metabolic equivalent; WC, waist circumference; HGS, handgrip strength; AMI, appendicular muscle mass index.

\section{Declarations}

\section{Ethics approval and consent to participate}

All subjects provided written, informed consent for their data to be used in the study, and the study protocol was approved by the Ethics Committee of Bengbu Medical College (Anhui, China; no. 2018045).

\section{Consent for Publication}

Not applicable.

Availability of data and material

Not applicable.

\section{Competing interests}

The authors of this paper have no competing interest.

\section{Funding}

This work was supported by the National Key R\&D Program of China [grant number 2017YFB1002204] and Anhui Science and Technology Department [grant number 18030801133].

\section{Authors' contributions}

Ting Sun: data analysis, manuscript drafting. ZuChang Ma: supervision, manuscript revision and editing. Lisheng Gao: manuscript editing. Hui Xie: data analysis, manuscript revision and editing.

\section{Acknowledgements}

The authors thank Lan Xie and Lin Liu at the Community Health Service Centre for administrative help.

\section{References}

1. Zeng YL, Hu XY, Xie LL, Han ZL, Zuo Y, Yang M. The Prevalence of Sarcopenia in Chinese Elderly Nursing Home Residents: A Comparison of 4 Diagnostic Criteria. Journal of the American Medical Directors Association [Article]. 2018 Aug;19(8):690-5.

2. Chen LK, Woo J, Assantachai P, Auyeung TW, Chou MY, lijima K, et al. Asian Working Group for Sarcopenia: 2019 Consensus Update on Sarcopenia Diagnosis and Treatment. J Am Med Dir Assoc. 2020 Mar;21(3):300-7 e2. 
3. Davies B, Garcia F, Ara I, Artalejo FR, Rodriguez-Manas L, Walter S. Relationship Between Sarcopenia and Frailty in the Toledo Study of Healthy Aging: A Population Based Cross-Sectional Study. Journal of the American Medical Directors Association [Article]. 2018 Apr;19(4):282-6.

4. Yoshimura N, Muraki S, Oka H, lidaka T, Kodama R, Kawaguchi H, et al. Is osteoporosis a predictor for future sarcopenia or vice versa? Fouryear observations between the second and third ROAD study surveys. Osteoporos Int. 2017 Jan;28(1):189-99.

5. Iwasaki M, Kimura Y, Ogawa H, Wada T, Sakamoto R, Ishimoto Y, et al. The association between dentition status and sarcopenia in Japanese adults aged 75 years. J Oral Rehabil. 2017 Jan;44(1):51-8.

6. Tanishima S, Hagino H, Matsumoto H, Tanimura C, Nagashima H. Association between sarcopenia and low back pain in local residents prospective cohort study from the GAINA study. Bmc Musculoskeletal Disorders. 2017 Nov; 18.

7. Wang TT, Feng X, Zhou JJ, Gong HY, Xia S, Wei Q, et al. Type 2 diabetes mellitus is associated with increased risks of sarcopenia and presarcopenia in Chinese elderly. Scientific Reports. 2016 Dec;6.

8. Wang H, Hai S, Liu YX, Cao L, Liu Y, Liu P, et al. Association between depressive symptoms and sarcopenia in older Chinese communitydwelling individuals. Clin Interv Aging. 2018;13:1605-11.

9. Hu XY, Jiang JJ, Wang HZ, Zhang L, Dong BR, Yang M. Association between sleep duration and sarcopenia among community-dwelling older adults A cross-sectional study. Medicine. 2017 Mar;96(10).

10. Han PP, Yu HR, Ma YX, Kang L, Fu LY, Jia LY, et al. The increased risk of sarcopenia in patients with cardiovascular risk factors in SuburbDwelling older Chinese using the AWGS definition. Scientific Reports. 2017 Aug;7.

11. Hai S, Wang H, Cao L, Liu P, Zhou JH, Yang Y, et al. Association between sarcopenia with lifestyle and family function among communitydwelling Chinese aged 60 years and older. Bmc Geriatrics. 2017 Aug;17.

12. Hai S, Cao L, Wang H, Zhou JH, Liu P, Yang Y, et al. Association between sarcopenia and nutritional status and physical activity among community-dwelling Chinese adults aged 60 years and older. Geriatrics Gerontology International. 2017 Nov;17(11):1959-66.

13. Woo J, Leung J, Morley JE. Defining Sarcopenia in Terms of Incident Adverse Outcomes. J Am Med Dir Assoc. 2015 Mar;16(3):247-52.

14. Kuo YH, Wang TF, Liu LK, Lee WJ, Peng LN, Chen LK. Epidemiology of Sarcopenia and Factors Associated With It Among Community-Dwelling Older Adults in Taiwan. Am J Med Sci. 2019 Aug;357(2):124-33.

15. Munakata M. Brachial-ankle pulse wave velocity in the measurement of arterial stiffness: recent evidence and clinical applications. Current hypertension reviews 2014. 2014;10(1):49-57.

16. Yamashina A, Tomiyama H, Arai T, Hirose K, Koji Y, Hirayama Y, et al. Brachial-ankle pulse wave velocity as a marker of atherosclerotic vascular damage and cardiovascular risk. Hypertens Res. 2003 Aug;26(8):615-22.

17. Kario AY K KK. Guidelines for noninvasive vascular function test(JCS2013). Japanese. 2013(01).

18. Hu PPQG, Luo SS. Research progress of sarcopenia and arteriosclerosis. Geriatr Health Care. 2020;26(1):141-4.

19. Shuang T, Fu M, Yang GD, Wu LY, Wang R. The interaction of IGF-1/IGF-1R and hydrogen sulfide on the proliferation of mouse primary vascular smooth muscle cells. Biochem Pharmacol. 2018 Mar;149:143-52.

20. Bahat G, Tufan A, Tufan F, Kilic C, Akpinar TS, Kose M, et al. Cut-off points to identify sarcopenia according to European Working Group on Sarcopenia in Older People (EWGSOP) definition. Clinical Nutrition. 2016 Dec;35(6):1557-63.

21. Yamanashi H, Kulkarni B, Edwards T, Kinra S, Koyamatsu J, Nagayoshi M, et al. Association between atherosclerosis and handgrip strength in non-hypertensive populations in India and Japan. Geriatrics \& Gerontology International. [Article]. 2018 Jul;18(7):1071-8.

22. van Dijk SC, Swart KMA, Ham AC, Enneman AW, Van Wijngaarden JP, Feskens EJ, et al. Physical fitness, activity and hand-grip strength are not associated with arterial stiffness in older individuals. Journal of Nutrition Health Aging [Article]. 2015 Aug;19(7):779-84.

23. Abbatecola AM, Chiodini P, Gallo C, Lakatta E, Sutton-Tyrrell K, Tylavsky FA, et al. Pulse wave velocity is associated with muscle mass decline: Health ABC study. Age. 2012 Apr;34(2):469-78.

24. Hida T, Imagama S, Ando K, Kobayashi K, Muramoto A, Ito K, et al. Sarcopenia and physical function are associated with inflammation and arteriosclerosis in community-dwelling people: The Yakumo study. Mod Rheumatol. 2018;28(2):345-50.

25. Kalinkovich A, Livshits G. Sarcopenic obesity or obese sarcopenia: A cross talk between age-associated adipose tissue and skeletal muscle inflammation as a main mechanism of the pathogenesis. Ageing Research Reviews. 2017 May;35:200-21.

26. Huang Y-q, Li J, Chen J-y, Zhou Y-I, Cai A-p, Huang C, et al. The Association of Circulating MiR29b and Interleukin-6 with Subclinical Atherosclerosis. Cellular Physiology and Biochemistry. 2017 2017;44(4):1537-44.

27. Stancel N, Chen CC, Ke LY, Chu CS, Lu J, Sawamura T, et al. Interplay between CRP, Atherogenic LDL, and LOX-1 and Its Potential Role in the Pathogenesis of Atherosclerosis. Clinical Chemistry. 2016 Feb;62(2):320-7.

28. Lee YH, Jung KS, Kim SU, Yoon HJ, Yun YJ, Lee BW, et al. Sarcopaenia is associated with NAFLD independently of obesity and insulin resistance: Nationwide surveys (KNHANES 2008-2011). J Hepatol. 2015 Aug;63(2):486-93.

29. Luevano-Contreras C, Garay-Sevilla ME, Wrobel K, Malacara JM, Wrobel K. Dietary advanced glycation end products restriction diminishes inflammation markers and oxidative stress in patients with type 2 diabetes mellitus. Journal of Clinical Biochemistry Nutrition. 2013

Page $8 / 10$ 
Jan;52(1):22-6.

30. Miyabe M, Ohashi K, Shibata R, Uemura Y, Ogura Y, Yuasa D, et al. Muscle-derived follistatin-like 1 functions to reduce neointimal formation after vascular injury. Cardiovascular Research. 2014 Jul;103(1):111-20.

\section{Figures}

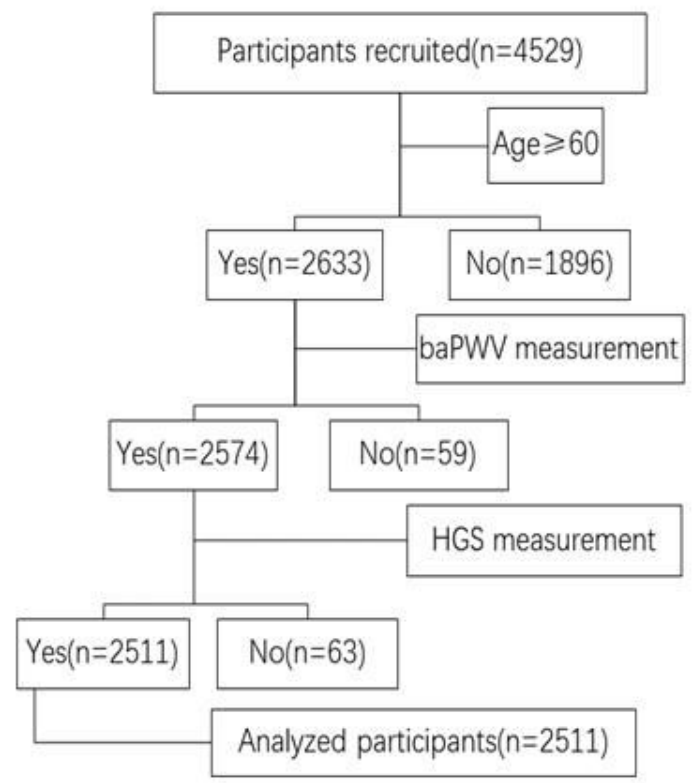

Figure 1. Flow diagram of participant recruitment and screening . baPWV, brachial-ankle pulse wave velocity: HGS, handgrip strength.

\section{Figure 1}

Flow diagram of participant recruitment and screening. belaWV. brachial-ankle pulse wave velocity; $11 \mathrm{GS}$. handerm strength.
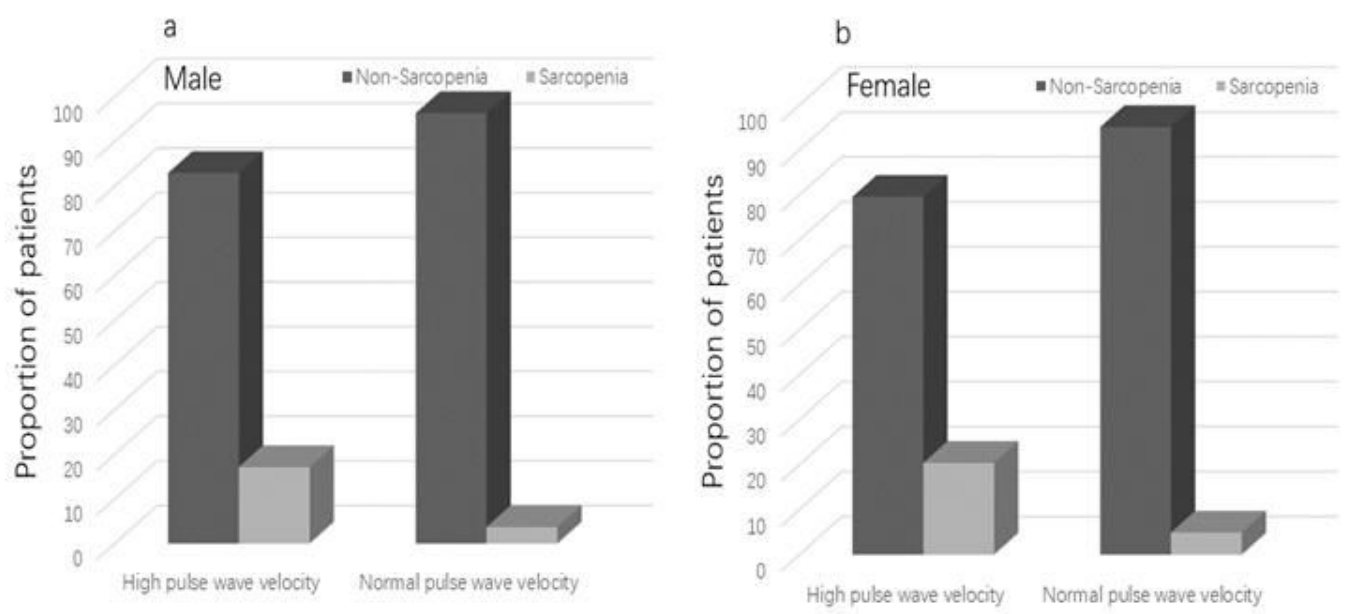

Figure 2 The proportions of subjects with and without sarcopenia in high and normal pulse wave velocity groups among males (a) and females (b). Chi-squared tests were used to detect the differences in the distributions of sarcopenia in the high and normal pulse wave velocity groups, both $P<0.001$. 


\section{Figure 2}

The proportions of subjects with and without sarcopenia in high and normal pulse wave velocity groups among males (a) and females (b). Chisquared tests were used to detect the differences in the distributions of sarcopenia in the high and normal pulse wave velocity groups. both $P<0.001$.
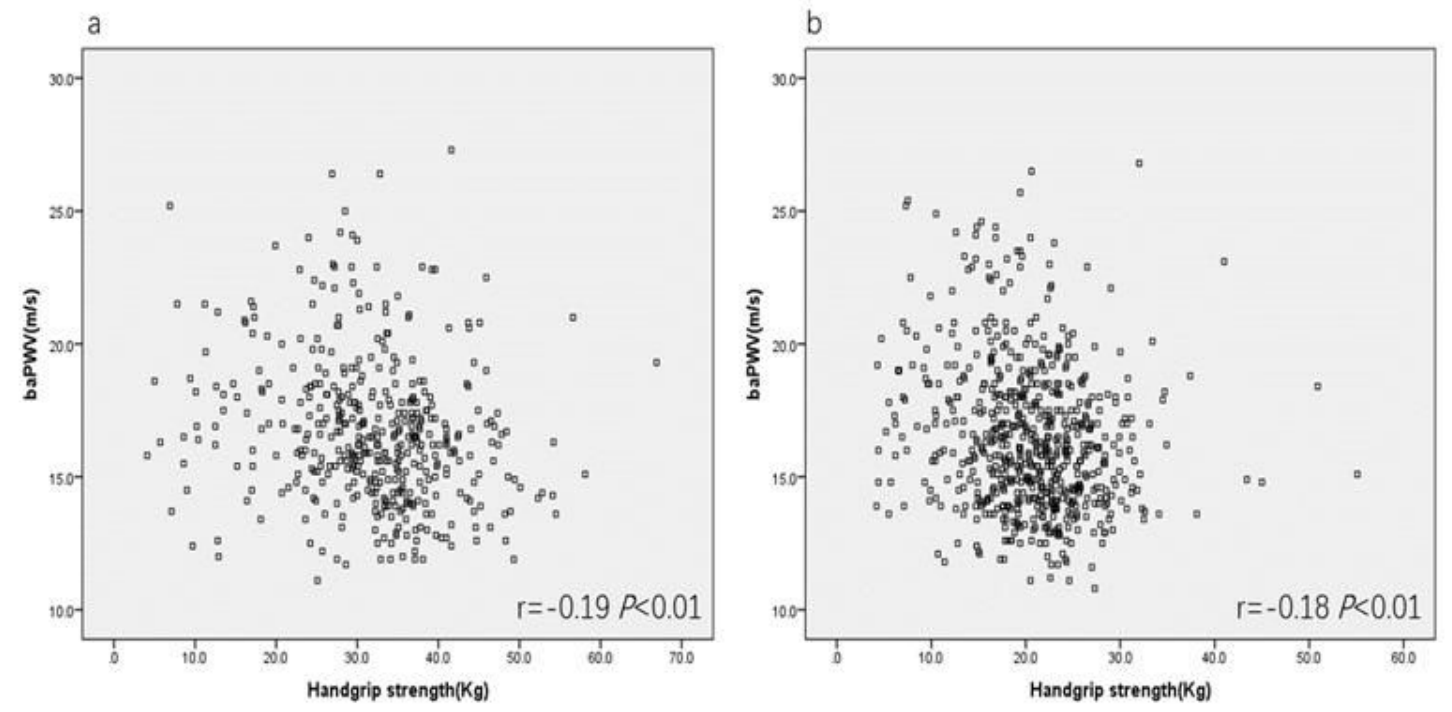

Figure 3. Scatter plot of handgrip strength against baPWV in male (a) and female (b) subjects, baPWV: brachial-ankle pulse wave velocity.

\section{Figure 3}

Scatter plot of handgrip strength against baPWV in male (a) and female (b) subjects, baPWV: brachial-ankle pulse wave velocity. 